\title{
Molecular identification of sulfadoxine-pyrimethamine resistance in malaria infected women who received intermittent preventive treatment in the Democratic Republic of Congo
}

\author{
Emrah Ruh ${ }^{1 *} \mathbb{0}$, Jean Paul Bateko ${ }^{1,2}$, Turgut Imir ${ }^{1}$ and Aysegul Taylan-Ozkan ${ }^{1,3}$
}

\begin{abstract}
Background: Point mutations in Plasmodium falciparum dihydrofolate reductase (Pfdhfr) and dihydropteroate synthase (Pfdhps) genes which confer resistance to sulfadoxine-pyrimethamine (SP) occur at increasing rates. The present study aimed to identify Pfdhfr and Pfdhps mutations in P. falciparum isolates recovered from women who received two doses of SP during pregnancy in Bandundu, the Democratic Republic of Congo (DRC).

Methods: A total of 48 women with confirmed P. falciparum infection were enrolled in the study. Finger-prick blood samples that were collected on filter paper at the time of delivery were used for DNA isolation. Pfdhfr and Pfdhps genes were amplified by a nested PCR protocol. DNA sequencing was performed on both strands, and the point mutations were analysed.

Results: All of the 48 (100.0\%) P. falciparum isolates carried at least one polymorphism in both genes. The wild-type haplotypes of Pfdhfr (CNCSI [C50, N51, C59, S108, 1164]) and Pfdhps (SAKAA [S436, A437, K540, A581, A613]) were not observed in the study. In Pfdhfr, N51I (85.4\%), C59R (60.4\%), and S108N (100.0\%) polymorphisms were detected. Triple mutation (CIRNI) (mutant amino acids are underlined) was the most prevalent (47.9\%) Pfdhfr haplotype. In the study, all P. falciparum isolates (100.0\%) harboured the A437G allele in Pfdhps gene. Also, K540E and A581G polymorphisms were observed in one (2.1\%) isolate. Single mutant haplotype (SGKAA) was detected in $97.9 \%$ of the isolates. Mutant Pfdhfr and Pfdhps allele combinations revealed quintuple (CICNI-SGEGA; 2.1\%), quadruple (CIRNI-SGKAA; 47.9\%), triple (CICNI-SGKAA; 35.4\%, CNRNI-SGKAA; 12.5\%), and double (CNCNI-SGKAA; $2.1 \%$ ) haplotypes.

Conclusions: In the study, the rate of SGEGA haplotype was low (2.1\%). Although K540E and A581G alleles are more common in Eastern Africa, a distinct lineage of SGEGA is also present in the DRC, which is located in Central Africa. This haplotype is associated with decreased efficacy of SP in pregnant women and infants, therefore, it should be carefully considered in the DRC and SP resistance should be routinely monitored.
\end{abstract}

Keywords: Plasmodium falciparum, Sulfadoxine-pyrimethamine, Drug resistance, Pfdhfr, Pfdhps, Democratic Republic of Congo

\footnotetext{
*Correspondence: emrah.ruh@neu.edu.tr

${ }^{1}$ Department of Medical Microbiology and Clinical Microbiology, Near

East University Faculty of Medicine, Near East Boulevard, 99138 Nicosia

Northern Cyprus

Full list of author information is available at the end of the article
} 


\section{Background}

The second highest rate of global malaria prevalence is documented in the Democratic Republic of Congo (DRC) [1]. Affecting a number of risk groups, malaria is also a major concern in pregnancy. More than half of the pregnant women at risk of malaria infection live in sub-Saharan Africa [2]. The infection leads to maternal anaemia and low birth weight (LBW), which results in infant mortality [2]. For this reason, the World Health Organization (WHO) recommends the administration of intermittent preventive treatment in pregnancy with sulfadoxinepyrimethamine (IPTp-SP) [3]. The WHO guidelines that were updated in 2013 ensure a minimum of three doses of SP in pregnancy [3].

In the DRC, approximately $97 \%$ of the population lives in areas with stable malaria transmission that lasts for 8-12 months of the year [4]. In the country, IPTp was adopted in 2003 and SP has been used to prevent malaria in pregnant women and the newborns. Nevertheless, IPTp coverage has been reported to be low. The percentage of women receiving at least two doses of SP only increased from 5 to 14\% over 2007-2013 period [4]. IPTp guidelines in the DRC were also revised in 2013 according to the WHO recommendations. The current target is administration of two and three doses of IPTp to a minimum of 60 and $30 \%$ of pregnant women, respectively [4].

Increasing resistance is of concern because this can limit the antimalarial activity of SP [5]. Previously in the DRC, SP treatment failure by day 28 was reported to be between 2 and $60 \%$. Thus, SP was replaced by artemisinin-based combination therapy (ACT) as the first-line treatment of malaria [2]. Point mutations in Plasmodium falciparum dihydrofolate reductase (Pfd$h f r$ ) and dihydropteroate synthase (Pfdhps) genes, encoding the related DHFR and DHPS enzymes in the folate-pathway, cause resistance against pyrimethamine and sulfadoxine, respectively [6]. Notably, SP resistance is initiated by single polymorphisms, and augmented by accumulation of mutations in Pfdhfr and Pfdhps genes [7]. Five mutations have been widely reported in Africa, which are N51I, C59R, S108N in Pfdhr; with A437G and K540E in Pfdhps gene [8]. In addition to K540E, A581G polymorphism in Pfdhps has been typically detected in East Africa [5]. Mutant Pfdhfr-Pfdhps haplotypes lead to high level resistance which is associated with decreased efficiency of IPTp-SP [5]. Moreover, the emergence of a sextuple mutant genotype, characterized with triple Pfdhfr (N51I + C59R + S108N) and triple Pfdhps (A437G + K540E + A581G) mutations, has been associated with reduced birth weight [9].

So far, a number of reports have shown the gene polymorphisms associated with SP resistance in the DRC [10-13]. However the molecular basis of resistance among women receiving IPTp-SP in the country remains unclear. Thus, the present study aimed to identify the point mutations in Pfdhfr and Pfdhps genes in P. falciparum isolates recovered from newly delivered women who received two doses of SP prophylaxis during pregnancy in the DRC.

\section{Methods \\ Study area, study population and collection of blood samples}

The DRC is one of the sub-Saharan African countries and located in the central region of the continent. This study was conducted in Bandundu city, which is situated in the west of the DRC. Between March and May (rainy season), and in September (end of the dry season) in 2014, a total of 250 consecutive women who gave birth at three different health centres in Bandundu were screened for malaria infection. All of the individuals received two doses of IPTp-SP at the 16th and 28th weeks of gestation. At the time of delivery, finger-prick blood samples were collected to perform microscopy and rapid diagnostic test (RDT). Additionally, blood samples were spotted and dried on Whatman filter paper. The presence of malaria infection was determined by microscopic examination of blood smears and evaluation of RDT results (SD Bioline Malaria Ag P.f./Pan). Of the 250 women, 48 were found positive for $P$. falciparum by both microscopy and RDT. Eventually, the 48 women [mean and median age: $27.6 \pm 6.5$ and 27.5 (16.0-38.0), respectively] with confirmed malaria infection were included in the study and their dried blood spots were used for further molecular analysis. Flow chart of the study protocol is given in Fig. 1.

\section{Ethical approval}

The ethical approval for the study was obtained from the Ankara Numune Training and Research Hospital Ethics Committee (Project no: E-14-286). Informed consent was collected from all participants.

\section{Molecular analysis of the resistance genes}

Molecular tests were performed by using the dried filter paper blood spots which were collected at the time of delivery and stored in sealed plastic bags at $4{ }^{\circ} \mathrm{C}$ (Fig. 1). DNA isolation from the dried blood spots was conducted according to the manufacturer's protocol (EURx GeneMATRIX Bio-Trace DNA Purification Kit). For amplification of Pfdhfr and Pfdhps genes, nested PCR assay was performed as described elsewhere [7]. Briefly, Pfdhfr gene was amplified by using the primers AMP-1 $\mathrm{F}$ and AMP-2R, which was followed by the nested reaction with primers M1 and M5. The 648-base pair fragment of the second PCR product covered the codons, 


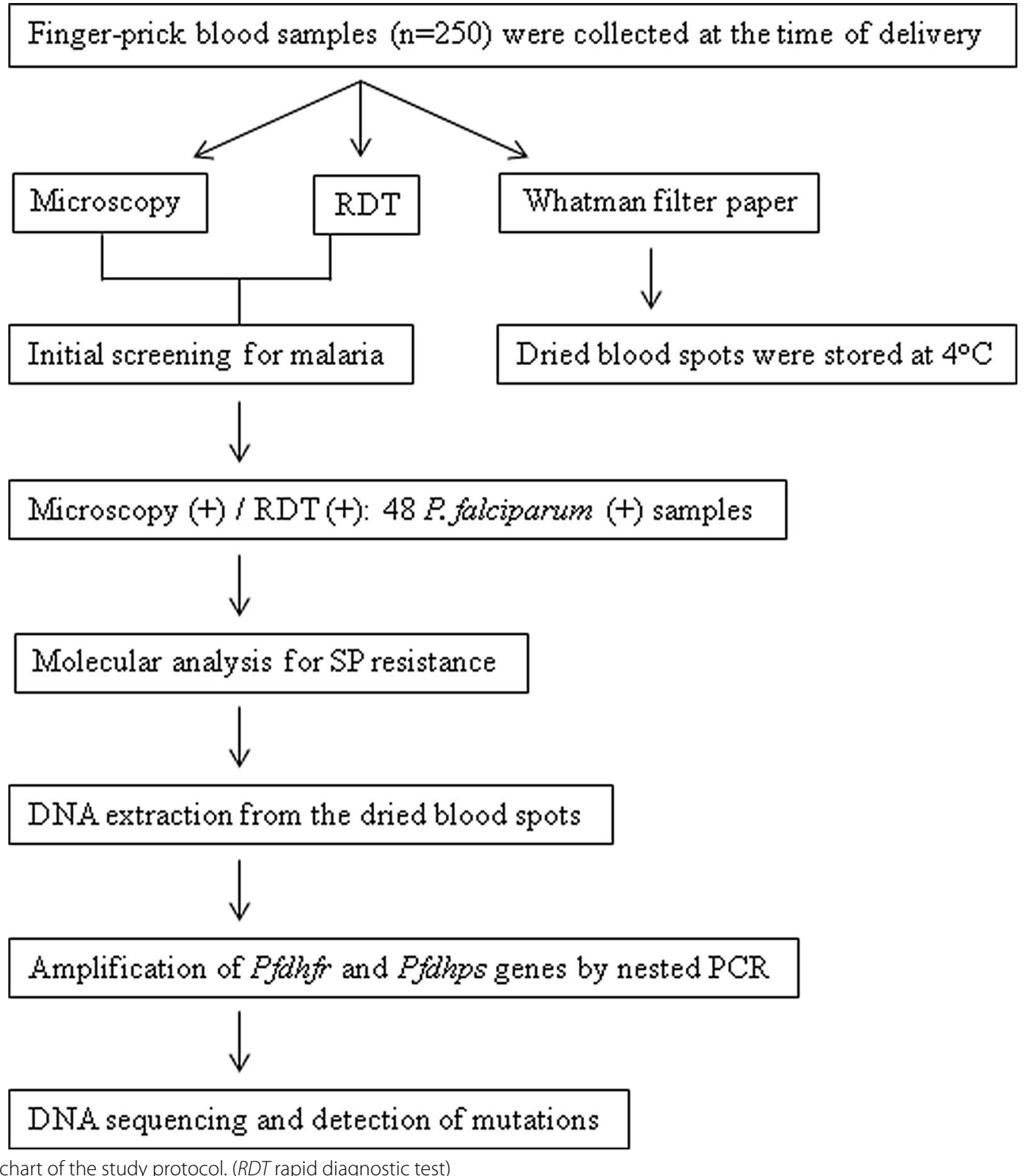

Fig. 1 Flow chart of the study protocol. (RDT rapid diagnostic test)

which are associated with pyrimethamine resistance. For Pfdhps gene, following the first round PCR with primers M3717F and 186R, the nested reaction was performed by using the primers Rc and Rd. The 728-bp fragment of the second amplicon covered the codons, which are related with resistance to sulfadoxine. Finally, the purified PCR products were sequenced in both strands and the single nucleotide polymorphisms (SNPs) were analysed [7]. The sequences of $P f d h f r$ and Pfdhps genes were submitted to GenBank under the accession numbers MG254624MG254671 and MG254672-MG254719, respectively.

\section{Results}

Pfdhfr gene polymorphisms

Of the 48 P. falciparum isolates sequenced, N51I ( $\mathrm{n}=41$, 85.4\%), C59R ( $\mathrm{n}=29,60.4 \%)$, and S108N ( $\mathrm{n}=48$, $100.0 \%)$ polymorphisms were detected. No mutation was observed at the codons 16, 50 and 164 (Table 1). The wild-type Pfdhfr haplotype (CNCSI [C50, N51, C59, S108, and I164]) was not identified in any of the isolates. Twenty-three (47.9\%) of 48 isolates were triple mutants (CIRNI) (SNPs are underlined). Number of double mutant haplotypes (CICNI, and CNRNI) was 18 (37.5\%) 
Table 1 Types of SNPs detected in Pfdhfr and Pfdhps genes among 48 Plasmodium falciparum isolates

\begin{tabular}{|c|c|c|c|}
\hline Mutant alleles in Pfdhfr gene ${ }^{a}$ & Number of the isolates, $\mathbf{n}(\%)$ & Mutant alleles in Pfdhps gene ${ }^{b}$ & Number of the isolates, $n(\%)$ \\
\hline N511 & $41 / 48(85.4)$ & A437G & $48 / 48(100.0)$ \\
\hline C59R & 29/48 (60.4) & $\mathrm{K} 540 \mathrm{E}$ & $1 / 48(2.1)$ \\
\hline $\mathrm{S} 108 \mathrm{~N}$ & $48 / 48(100.0)$ & A581G & $1 / 48(2.1)$ \\
\hline
\end{tabular}

a Pfdhfr polymorphisms: codon 51, asparagine to isoleucine; codon 59, cysteine to arginine; codon 108, serine to asparagine

b Pfdhps polymorphisms: codon 437, alanine to glycine; codon 540, lysine to glutamic acid; codon 581, alanine to glycine

and six (12.5\%), respectively; while one $(2.1 \%)$ isolate was single mutant (CNCNI) (Table 2).

\section{Pfdhps gene polymorphisms}

All P. falciparum isolates $(\mathrm{n}=48,100.0 \%)$ had A437G mutation. K540E and A581G polymorphisms were observed in one $(2.1 \%)$ isolate. No mutation was found at the codons 436 and 613 (Table 1). The wild-type Pfdhps haplotype (SAKAA [S436, A437, K540, A581, and A613]) was not detected in the study. In total, 47 (97.9\%) of the isolates were single mutant (SGKAA), and one $(2.1 \%)$ isolate had triple mutant haplotype (SGEGA) (Table 2).

\section{Combination of Pfdhfr and Pfdhps haplotypes}

In the study, all of the $P$. falciparum isolates $(n=48$, $100.0 \%$ ) carried at least one polymorphism in both genes. Mutant Pfdhfr and Pfdhps alleles detected in the 48 isolates were combined and five different PfdhfrPfdhps haplotypes were generated. Accordingly, one (2.1\%) isolate was classified as quintuple haplotype (double Pfdhfr with triple Pfdhps [CICNI-SGEGA]). In 23 (47.9\%) isolates, quadruple haplotype (triple $P f d h f r$ with single Pfdhps [CIRNI-SGKAA]) was identified. Triple haplotypes (double Pfdhfr with single Pfdhps [CICNI-

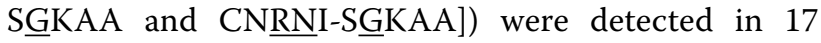
$(35.4 \%)$ and $6(12.5 \%)$ isolates, respectively. Finally, one (2.1\%) isolate was noted to be double haplotype (single Pfdhfr with single Pfdhps [CNCNI-SGKAA]). Types of Pfdhfr and Pfdhps allele combinations are summarized in Table 3.

\section{Discussion}

In the regions where malaria is endemic, $25 \%$ of pregnant women are estimated to be infected with Plasmodium parasites [14]. Therefore, maintenance of IPTp-SP in those regions is crucial for protecting both pregnant women and their infants against the adverse outcomes of malaria [3]. Considering the high prevalence of malaria infection in the DRC [1], pregnant women also constitute a risk group for the disease.

Despite the effect of IPTp-SP on birth weight was evaluated previously in the DRC [2], there is a lack of research addressing the molecular basis of SP resistance. Thus, the present study was carried out to identify the Pfdhfr and Pfdhps mutations in P. falciparum strains isolated from 48 individuals that received two doses of SP during pregnancy.

In the study, the highest percentage $(100.0 \%)$ of polymorphism in Pfdhfr gene was noted to be $S 108 \mathrm{~N}$, which was followed by N51I (85.4\%) and C59R (60.4\%) (Table 1). On the contrary, I164L mutation was not detected, and this finding is compatible with those reported elsewhere $[12,13]$. The prevalence of S108N was consistent with that of a previous report (99.1\%) from the DRC, however N51I and C59R rates found here differed from that study ( 97.9 and $80.7 \%$, respectively) [13]. On the contrary, the rate of C59R allele was consistent with that of another study (66\%) from the DRC [12]. Furthermore, findings of the present study support the hypothesis that pyrimethamine resistance is initiated by the codon 108 mutation, and improved by the additional polymorphisms at the other Pfdhfr codons [7].

Table 2 Types of mutant Pfdhfr and Pfdhps haplotypes among 48 Plasmodium falciparum isolates

\begin{tabular}{|c|c|c|c|c|}
\hline Haplotype & Pfdhfr gene ${ }^{a}$ & Number of the isolates, $n(\%)$ & Pfdhps gene ${ }^{\mathbf{b}}$ & Number of the isolates, $n(\%)$ \\
\hline Wild-type & CNCSI & $0 / 48(0.0)$ & SAKAA & $0 / 48(0.0)$ \\
\hline Triple & CIRNI & 23/48 (47.9) & SGEGA & $1 / 48(2.1)$ \\
\hline Double & CICNI & $18 / 48(37.5)$ & - & - \\
\hline Double & CNRNI & $6 / 48(12.5)$ & - & - \\
\hline Single & CNCNI & $1 / 48(2.1)$ & SGKAA & 47/48 (97.9) \\
\hline
\end{tabular}

a Pfdhfr wild-type haplotype (CNCSI [C50/N51/C59/S108/I164]); triple (511/59R/108N); double (511/108N, or 59R/108N); and single (108N) mutant

b Wild-type Pfdhps haplotype (SAKAA [S436/A437/K540/A581/A613]); triple (437G/540E/581G); and single (437G) mutant 
Table 3 Distribution of Pfdhfr and Pfdhps allele combinations among 48 Plasmodium falciparum isolates

\begin{tabular}{|c|c|c|}
\hline Haplotype & Pfdhfr-Pfdhps alleles ${ }^{a}$ & Number of the isolates, $n(\%)$ \\
\hline Quintuple & CICNI-SGEGA & $1 / 48(2.1)$ \\
\hline Quadruple & 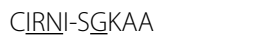 & 23/48 (47.9) \\
\hline Triple & CICNI-SGGKAA & $17 / 48(35.4)$ \\
\hline Triple & CNRNI-SGKKAA & $6 / 48(12.5)$ \\
\hline Double & CNCNII-SGKKAA & $1 / 48(2.1)$ \\
\hline
\end{tabular}

a Pfdhfr-Pfdhps allele combinations: quintuple (Pfdhfr 511/108N + Pfdfps 437G/540E/581G); quadruple (Pfdhfr 511/59R/108N + Pfdfps 437G); triple (Pfdhfr 511/108N + Pfdfps 437G); triple (Pfdhfr 59R/108N + Pfdfps 437G); and double (Pfdhfr 108N + Pfdfps 437G) haplotype

In the present study, $47.9 \%$ of the isolates were found to harbour the triple Pfdhfr mutation (CIRNI haplotype) (Table 2). This is lower than previously reported rates of $57.8-78.2 \%$ [11], however similar to the findings (46.2\%) of another study [10], which were both conducted in the DRC. The prevalence of $\mathrm{CICNI}(37.5 \%)$ and CNCNI (2.1\%) was comparable with those reported elsewhere; however the rate of CNRNI (12.5\%) in this study was found to be relatively higher [10]. In contrast with other studies where the rates of wild-type Pfdhfr were reported to be $1.0-7.6 \%$ [10-12], the CNCSI haplotype was not detected in the present study.

The Pfdhps A437G mutation was found in all (100.0\%) $P$. falciparum isolates sequenced (Table 1). The prevalence of this polymorphism was reported to be $93.1 \%$ [13] and 72\% [12] by former studies in the DRC. In contrast to the A437G allele, K540E in the present study was less prevalent (2.1\%) than previous findings (9.5\%) [13]. An increased rate of K540E polymorphism (67\%) was detected by another study in Rutshuru, the DRC [12], however this was attributed to the close proximity of the study area to Eastern Africa where SP resistance is high [15]. Therefore, the result presented here supports the previous studies that documented the rate of K540E to be less than $50 \%$ in the DRC [15]. On the contrary, the rate of A581G mutation in the present study $(2.1 \%)$ is different from the previous data, where it was reported to be higher than $10 \%$ in the DRC [15]. Also, no polymorphism was detected at the codon 613, which is consistent with earlier reports [12]. Taken together, the results presented here are in parallel with previous data which suggest that sulfadoxine resistance may be initiated by the mutations at codons 436 or 437 , and augmented by the additional polymorphisms at the other Pfdhps codons [7].

In this study, SGKAA carrying the single A437G mutant allele was the most prevalent (97.9\%) Pfdhps haplotype (Table 2). This result is higher than the recently documented percentage $(76 \%)$ in the DRC [5]. On the contrary, the rate of SGEGA haplotype
(437G/540E/581G) was low (2.1\%) in the present study. A similar percentage $(3.3 \%)$ was also noted by another study in the DRC [16]. The present result is consistent with the studies where double- and triple-mutant Pfdhps haplotypes were reported to be less common in Central Africa [5]. Recently, a higher percentage (8\%) of SGEGA has been detected in Kinshasa (located in the west of the DRC), but this was lower compared to the eastern countries, Tanzania (15\%) and Uganda (13\%) [5]. Of note, a study showed that SGEGA haplotypes from the DRC and the eastern countries (Malawi and Tanzania) separated into distinct lineages. These haplotypes lead to high levels of sulfadoxine resistance, thus compromising the efficacy of SP both in pregnant women and the infants [17]. Moreover, even the haplotypes in the DRC were found to be genetically and geographically clustered. Singlemutant haplotypes were shown to be more prevalent in the west, while the double- and triple-mutant haplotypes predominated in the east of the DRC [16]. Considering that the study setting, Bandundu city, is situated in the west of the DRC, this can explain the reason of low rate of SGEGA in contrast to the high number of Pfdhps single-mutant haplotypes found in the present survey. On the other hand, unlike the former studies which reported the prevalence of wild-type Pfdhps as high as $75.6 \%$ in the DRC [11], no sensitive strain (SAKAA) was found in the current study.

The combination of Pfdhfr-Pfdhps mutant alleles generated five different haplotypes in this study. CIRNISGKAA was found to be the most prevalent haplotype (47.9\%). This quadruple mutation was shown to occur at rates greater than $50 \%$ in African countries including the DRC. Also, this quadruple haplotype was associated with treatment failure [15].

The quintuple mutation (51I/59R/108N + 437G/540E) was previously associated with SP treatment failure and detected at rates of 27.1 and $43 \%$ in the studies from the DRC [10, 12]. Unlike those studies, here Pfdhfr $51 \mathrm{I} / 108 \mathrm{~N}$ allele was detected in combination with Pfdhps $437 \mathrm{G} / 540 \mathrm{E} / 581 \mathrm{G}$ in one $(2.1 \%)$ isolate, which generated another type of quintuple mutant haplotype (CICNISGEGA). This haplotype was also documented by a previous study from Tanzania [9]. Owing to the association between K540E and A581G mutations and worsened SP resistance in Eastern Africa [5], the mutant strains harbouring these alleles in the DRC should be carefully considered.

The triple mutant haplotypes (CICNI-S $\underline{\text { GKAA }}$ and CNRNI-SGKAA) were identified in 35.4 and $12.5 \%$ of the isolates, respectively. A study from India found the ANRNI-SGKAA allele combination (A: alanine at codon 16 of $P f d h f r$ ) in $0.92 \%$ of their isolates, and related this haplotype with low-level SP resistance [7]. Lastly, 
the mutant allele combination (CNCNI-SGKAA) was detected at low levels $(2.1 \%)$ in the present study. This result is lower than that reported for double mutant haplotype (16.7\%) in the DRC [10]. Similar to the single mutant allele in Pfdhfr or Pfdhps, the double mutant haplotype seems to occur at the initial stage of SP resistance [7].

In the present study, no Pfdhfr-Pfdhps sextuple mutant was detected, which is a promising result. Previously, sextuple haplotypes were associated with reduced birth weights [9]. Furthermore, newborns of women with the sextuple mutant haplotypes were found to have lower birth weights compared to those born to mothers with less mutated parasite infections [9]. Due to the lack of birth weight data in the study, no assessment was done between the mutation levels and the birth outcomes. Another limitation of this study is the relatively low number of participants. Yet, the mutations in both $P f d-$ $h f r$ and Pfdhps genes were confirmed by DNA sequencing in all $48(100.0 \%)$ P. falciparum isolates. The study results also indicate that the SGEGA haplotype is present in the DRC. Considering that the SGEGA haplotypes are associated with decreased IPTp-SP outcomes [17], these mutations should be carefully monitored in the DRC.

\section{Conclusions}

In the present study, the mutant Pfdhfr and Pfdhps haplotypes were identified in all of the $P$. falciparum strains isolated from 48 women at the time of delivery. The quadruple haplotype (CIRNI-SGKAA) which is common in Western Africa was also predominant in this study. The rate of quintuple haplotype (CICNI-SGEGA) was found to be very low. Although K540E and A581G alleles are more common in Eastern Africa, a distinct lineage of SGEGA is also present in the DRC. Therefore, despite the low levels found in this study, the triple Pfdhps mutation should be given particular consideration and SP resistance should be routinely monitored in the DRC. To the authors' knowledge, this is the first report from the DRC that addressed the molecular determinants of SP resistance in pregnancy. This study would provide a basis for future research to evaluate the correlation of mutation levels and the clinical outcomes of IPTp-SP in the DRC.

\section{Authors' contributions}

ATO, ER and TI conceived the study. JPB collected the samples and data. ER, JPB and ATO carried out interpretation of test results. ER and ATO drafted the manuscript. TI and JPB critically revised the manuscript for intellectual content. All authors read and approved the final manuscript.

\section{Author details}

${ }^{1}$ Department of Medical Microbiology and Clinical Microbiology, Near East University Faculty of Medicine, Near East Boulevard, 99138 Nicosia, Northern Cyprus. ${ }^{2}$ Department of Medical Biology, Higher Institute of Medical Technology, Bandundu, Democratic Republic of Congo. ${ }^{3}$ Department of Medical Microbiology, Hitit University Faculty of Medicine, Corum, Turkey.

\section{Acknowledgements}

The authors are grateful to the DRC Ministry of Health for their collaboration in the study. The authors would also like to thank Dr. Humen Cebbari from BM Labosis company (Ankara, Turkey) for his contributions to the molecular tests conducted in the study. This study was presented at the International Symposium on Parasitic Zoonoses (oral presentation no: PSS-07), which was held in Antalya, Turkey, 18-19 November, 2016.

\section{Competing interests}

The authors declare that they have no competing interests.

\section{Availability of data and materials}

All data generated or analysed during this study are included in this published article.

\section{Consent for publication}

Not applicable.

\section{Ethics approval and consent to participate}

The ethical approval for the study was obtained from the Ankara Numune Training and Research Hospital Ethics Committee (Project no: E-14-286). Informed consent was collected from all participants.

\section{Funding}

This study was supported by Near East University, Hitit University (Grant No: TIP19002.14.005) and Deniz Medical.

\section{Publisher's Note}

Springer Nature remains neutral with regard to jurisdictional claims in published maps and institutional affiliations.

Received: 3 November 2017 Accepted: 23 December 2017

Published online: 09 January 2018

\section{References}

1. ACTwatch Group, Mpanya G, Tshefu A, Likwela JL. The malaria testing and treatment market in Kinshasa, Democratic Republic of the Congo, 2013. Malar J. 2017;16:94.

2. Likwela JL, D'Alessandro U, Lokwa BL, Meuris S, Dramaix MW. Sulfadoxinepyrimethamine resistance and intermittent preventive treatment during pregnancy: a retrospective analysis of birth weight data in the Democratic Republic of Congo (DRC). Trop Med Int Health. 2012;17:322-9.

3. WHO. Policy brief for the implementation of intermittent preventive treatment of malaria in pregnancy using sulfadoxine-pyrimethamine (IPTp-SP). Geneva: World Health Organization; 2013 (revised January 2014).

4. President's malaria initiative. Democratic Republic of the Congo. Malaria Operational Plan FY; 2017.

5. Baraka V, Delgado-Ratto C, Nag S, Ishengoma DS, Madebe RA, Mavoko HM, et al. Different origin and dispersal of sulfadoxine-resistant Plasmodium falciparum haplotypes between Eastern Africa and Democratic Republic of Congo. Int J Antimicrob Agents. 2017;49:456-64.

6. Sutherland CJ, Fifer H, Pearce RJ, bin Reza F, Nicholas M, Haustein T, et al. Novel pfdhps haplotypes among imported cases of Plasmodium falciparum malaria in the United Kingdom. Antimicrob Agents Chemother. 2009:53:3405-10.

7. Sharma D, Lather M, Mallick PK, Adak T, Dang AS, Valecha N, et al. Polymorphism in drug resistance genes dihydrofolate reductase and dihydropteroate synthase in Plasmodium falciparum in some states of India. Parasit Vectors. 2015;8:471.

8. Heinberg A, Kirkman L. The molecular basis of antifolate resistance in Plasmodium falciparum: looking beyond point mutations. Ann NY Acad Sci. 2015;1342:10-8.

9. Minja DT, Schmiegelow C, Mmbando B, Boström S, Oesterholt M, Magistrado $\mathrm{P}$, et al. Plasmodium falciparum mutant haplotype infection during pregnancy associated with reduced birthweight, Tanzania. Emerg Infect Dis. 2013;19(9):1446-54. 
10. Swarthout TD, van den Broek IV, Kayembe G, Montgomery J, Pota H, Roper C. Artesunate + amodiaquine and artesunate + sulphadoxinepyrimethamine for treatment of uncomplicated malaria in Democratic Republic of Congo: a clinical trial with determination of sulphadoxine and pyrimethamine-resistant haplotypes. Trop Med Int Health. 2006;11:1503-11.

11. Cohuet S, Bonnet M, Van Herp M, Van Overmeir C, D'Alessandro U, Guthmann JP. Short report: molecular markers associated with Plasmodium falciparum resistance to sulfadoxine-pyrimethamine in the Democratic Republic of Congo. Am J Trop Med Hyg. 2006;75:152-4.

12. Alker AP, Kazadi WM, Kutelemeni AK, Bloland PB, Tshefu AK, Meshnick SR. dhfr and dhps genotype and sulfadoxine-pyrimethamine treatment failure in children with falciparum malaria in the Democratic Republic of Congo. Trop Med Int Health. 2008;13:1384-91.

13. Mobula L, Lilley B, Tshefu AK, Rosenthal PJ. Resistance-mediating polymorphisms in Plasmodium falciparum infections in Kinshasa, Democratic Republic of the Congo. Am J Trop Med Hyg. 2009;80:555-8.
14. Matangila JR, Lufuluabo J, Ibalanky AL, da Luz RAI, Van Lutumba JP, Geertruyden JP. Asymptomatic Plasmodium falciparum infection is associated with anaemia in pregnancy and can be more cost-effectively detected by rapid diagnostic test than by microscopy in Kinshasa, Democratic Republic of the Congo. Malar J. 2014;13:132.

15. Naidoo I, Roper C. Mapping 'partially resistant', 'fully resistant', and 'super resistant' malaria. Trends Parasitol. 2013;29:505-15.

16. Taylor SM, Antonia AL, Parobek CM, Juliano JJ, Janko M, Emch M, et al. Plasmodium falciparum sulfadoxine resistance is geographically and genetically clustered within the DR Congo. Sci Rep. 2013;3:1165.

17. Taylor SM, Antonia AL, Harrington WE, Goheen MM, Mwapasa V, Chaluluka E, et al. Independent lineages of highly sulfadoxine-resistant Plasmodium falciparum haplotypes, eastern Africa. Emerg Infect Dis. 2014;20:1140-8.

\section{Submit your next manuscript to BioMed Central and we will help you at every step:}

- We accept pre-submission inquiries

- Our selector tool helps you to find the most relevant journal

- We provide round the clock customer support

- Convenient online submission

- Thorough peer review

- Inclusion in PubMed and all major indexing services

- Maximum visibility for your research

Submit your manuscript at www.biomedcentral com/submit 